\title{
65 years of type 1 diabetes with total cessation of insulin therapy due to an IGF-2 producing tumour
}

\section{K Mohammed 1,2, M Aye $^{1,2}$.}

1. Department of Diabetes, Endocrinology and Metabolism, Hull Royal Infirmary,

2. Hull York Medical School. Kingston upon Hull HU3 2JZ, UK

A 73 year old patient with a 65 year history of type 1 diabetes mellitus developed recurrent hypoglycaemia. As a retired scientist, he maintained meticulous glycaemic control and kept copious records. He was on a basal-bolus regime with twice daily Insulatard and NovoRapid three times daily. $\mathrm{He}$ had numerous hypoglycaemic episodes despite a gradual reduction in insulin dose and a marked increase in dietary carbohydrate intake. Hypoglycaemic episodes occurred without any clear relationship to food or physical activity and with no consistent pattern [Fig. 1]. He lost approximately $3 \mathrm{~kg}$ in weight over the preceding year. He does not drink alcohol. His injection sites were reviewed and showed no lipodystrophy. His renal and liver functions were normal. His blood tests showed normochoromic normocytic anaemia, normal renal, adrenal and thyroid function. Coeliac disease was excluded and faecal elastase was normal.

As he continued to have neuroglycopenic hypoglycaemia despite minimal insulin, he was electively hospitalised to undergo a monitored withdrawal of insulin and a 72-hour fast. He developed no ketosis despite being off insulin and had spontaneous hypoglycaemia fulfilling Whipple's triad. His blood glucose was $1.8 \mathrm{mmol} / \mathrm{L}$, Serum insulin $<10 \mathrm{pmol} / \mathrm{L}, \mathrm{C}$-peptide $<94 \mathrm{pmol} / \mathrm{L}$. No insulin antibodies were detected. His Serum IGF-1 and IGF-BP3 were normal but IGF-2 was elevated at $87.0 \mathrm{nmol} / \mathrm{L}$. These results were consistent with non-islet cell tumour hypoglycemia. His chest x-ray showed elevation of the right hemidiaphragm. A CT scan of his chest, thorax and abdomen was performed which showed a $26 \mathrm{~cm}$ retrosternal mass [Fig. 2]

He underwent a successful complete excision of the solid fibrous tumour following which he immediately became hyperglycaemic and was placed back on twice daily Insulatard and Novorapid three times daily with no recurrence of hypoglycaemia.

The histology showed a fibrous based tumour measured $26 \mathrm{~cm}$ by $20 \mathrm{~cm}$ with a thickness of $1.3 \mathrm{~cm}$. The specimen was predominantly a spindle cell tumour of variable cellularity and of a 'pattern-less" pattern showing an intimate relationship to the surrounding fibrous stroma. The immunohistochemistry of the tumour showed positivity for CD34, CD39 and vimentin.
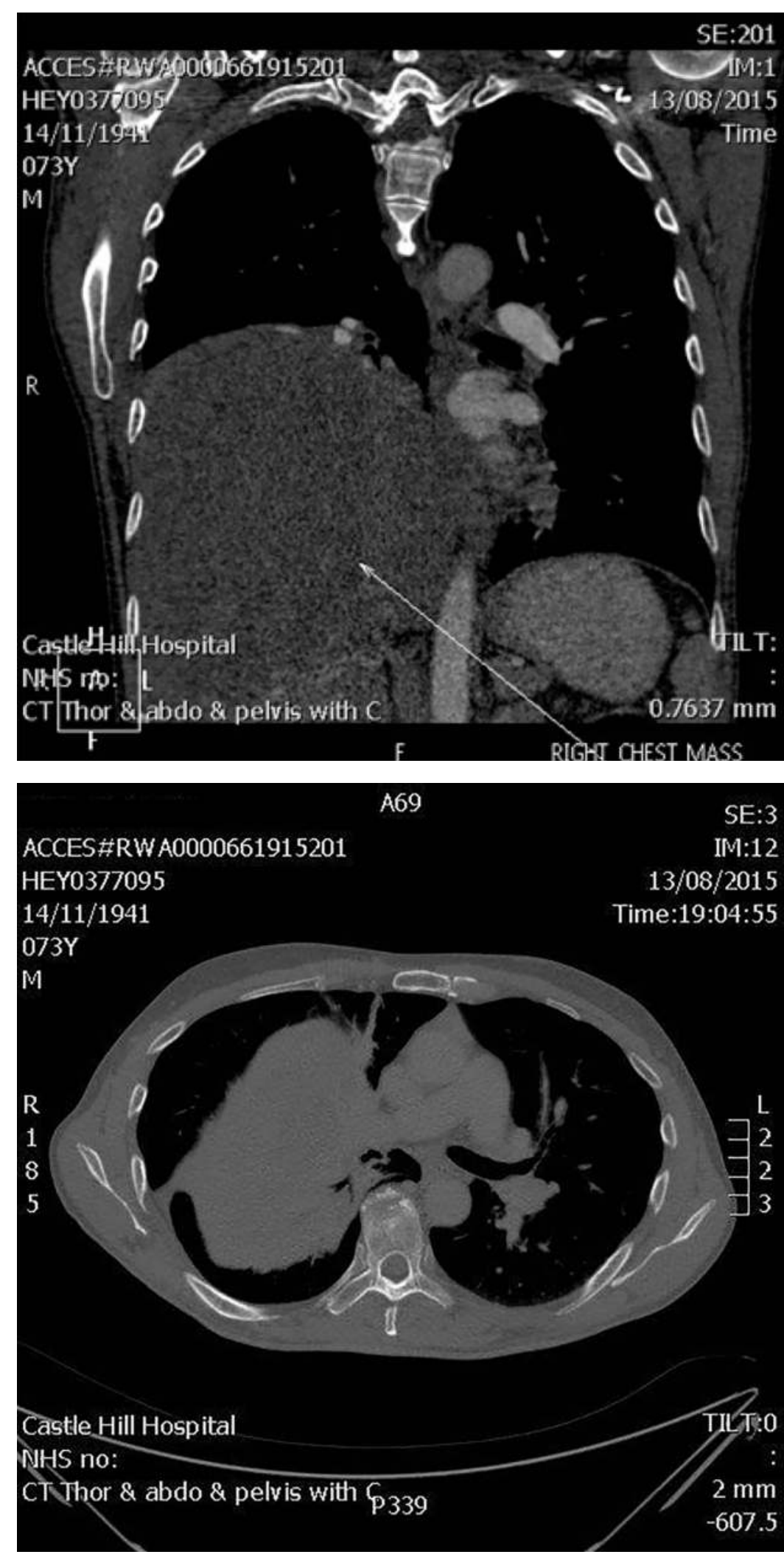

Figure 2. CT scan of the fibrous tumour.

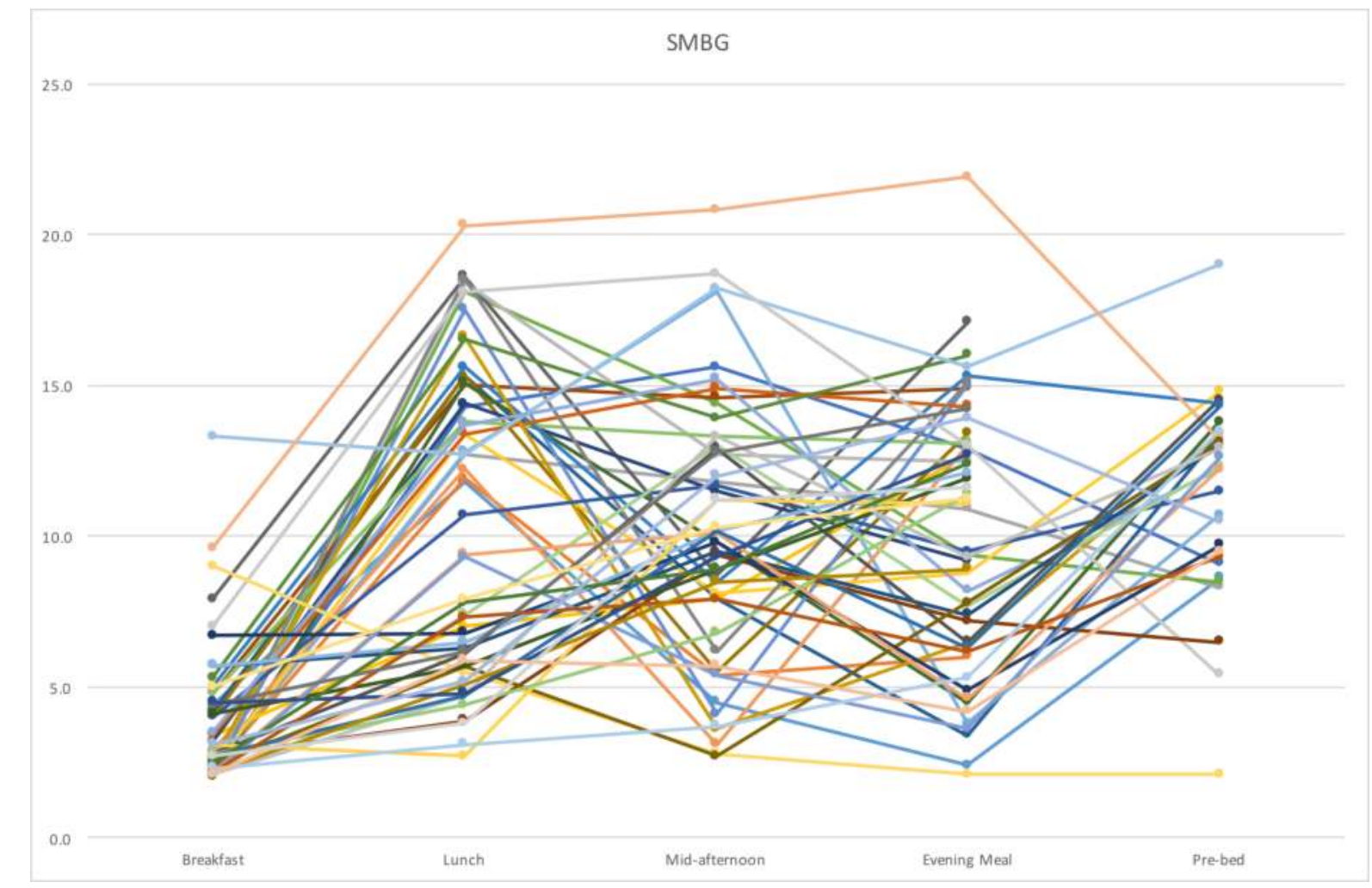

Our case is unique as it is the first reported case of 'remission' of longstanding type 1 diabetes due to an IGF-2 producing tumour. Surgery was justified despite the tumour being benign: the benefit of insulinfree 'remission' was far outweighed by the risks of recurrent hypoglycaemia. Unexplained hypoglycaemia warrants careful and structured evaluation even in patients who have established diabetes and in this case led to the diagnosis of this unique case.

Figure 1. Self-monitored glucose readings over a 2 month period. 